Check for updates

Cite this: RSC Adv., 2022, 12, 4187

Received 23rd December 2021 Accepted 21st January 2022

DOI: $10.1039 / \mathrm{d} 1 \mathrm{ra09298g}$

rsc.li/rsc-advances

\section{Diastereoselective synthesis of new zwitterionic bicyclic lactams, scaffolds for construction of 2- substituted-4-hydroxy piperidine and its pipecolic acid derivatives $\uparrow$}

\author{
Enrique Reyes-Bravo, ${ }^{a}$ Dino Gnecco, ${ }^{a}$ Jorge R. Juárez, (DD a María L. Orea, ${ }^{a}$ \\ Sylvain Bernès, (D) ${ }^{b}$ David M. Aparicio*a and Joel L. Terán (DD *a
}

\begin{abstract}
The synthesis of new chiral highly functionalized zwitterionic bicyclic lactams starting from acyclic $\beta$-enaminoesters derived from $(R)-(-)$-2-phenylglycinol is described. The key step involved an intramolecular non-classical Corey-Chaykovsky ring-closing reaction of the corresponding sulfonium salts derived from $\beta$-enaminoesters. This methodology permits the generation of two or three new stereogenic centers with high diastereoselectivity. The utility of these intermediates was demonstrated by the stereocontrolled total synthesis of cis-4-hydroxy-2-methyl piperidine and its corresponding pipecolic acid derivative.
\end{abstract}

Stereocontrolled synthesis of polysubstituted piperidines is a very important field in organic synthesis due to the great variety of piperidine-moiety-containing drugs, making it the most prevalent nitrogen heterocycle in approved drugs; ${ }^{1}$ therefore, their synthesis has been the focus of considerable attention.

In this sense, chiral non-racemic bicyclic lactams derived from $\beta$-amino alcohols are commonly considered useful starting materials for preparing these substituted piperidine drugs. ${ }^{2}$ One of the most effective methodologies for the synthesis of these bicyclic lactams is via an aza-annulation of $\beta$-enaminoesters ${ }^{3}$ derived from suitable $\beta$-amino alcohols. ${ }^{4}$

We recently reported the preparation of new cyclic zwitterionic intermediates via an intramolecular non-classical CoreyChaykovsky ring-closing reaction. Their utility was demonstrated by the synthesis of stereoisomers of $\sigma^{1}$-receptor agonists trozamicol $^{5}$ or the synthesis of (+)- and (-)-Geissman-Waiss lactone (Scheme 1). ${ }^{6}$

Considering this precedent and highlighting the utility of cyclic zwitterionic intermediates, we present a new versatile methodology to access new chiral zwitterionic non-racemic bicyclic lactams starting from acyclic $\beta$-aminoesters derived

${ }^{a}$ Centro de Quíinstituto de Cienciasmica, Instituto de Ciencias, Benemérita Universidad Autónoma de Puebla, Edif. IC-9 Complejo de Ciencias C.U., 72570 Puebla, Mexico.E-mail: joel.teran@correo.buap.mx

${ }^{b}$ Instituto de Física, Benemérita Universidad Autónoma de Puebla, 1IF2(aka 110-B), Lab. 102, C.U., 72570 Puebla, Mexico

$\dagger$ Electronic supplementary information (ESI) available. CCDC 2125364-2125371. For ESI and crystallographic data in CIF or other electronic format see DOI: 10.1039/d1ra09298g from $(R)-(-)$-2-phenylglycinol and its utility in the diastereoselective synthesis of 2-substituted-4-hydroxy piperidines.

We commence our finding by synthesizing of a set of $\beta$ enaminoesters coming from $(R)-(-)$-2-phenylglycinol, which were prepared following the traditional methodologies, directly condensation of chiral amine with $\beta$-dicarbonyl compound ${ }^{7}$ or by addition to a corresponding alkyne. ${ }^{8}$ All enamino esters were obtained as an inseparable $E: Z$ isomeric mixture and, these results are summarized below (Fig. 1).

With a set of chiral $\beta$-enaminoesters in hand, next these compounds were condensed with bromo acetyl bromide, ${ }^{9}$ affording the desirable $N$-acyl oxazolidine with the formation of a new stereogenic center at the hemiaminal position in good to excellent diastereomeric ratio. The stereochemistry at the new stereogenic center of the major diastereoisomer was determined from its X-ray diffraction analysis of $N$-acyl oxazolidine 10 as $(2 R),{ }^{10}$ therefore we assumed that this is the

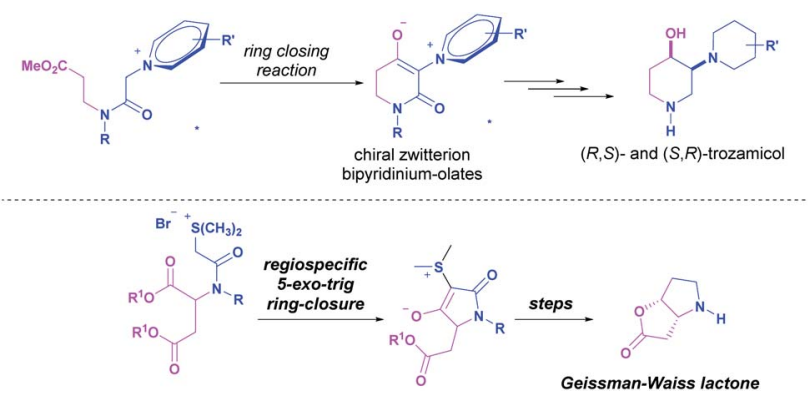

Scheme 1 Previous reports in the synthesis and utility of chiral cyclic zwitterionic intermediates. 


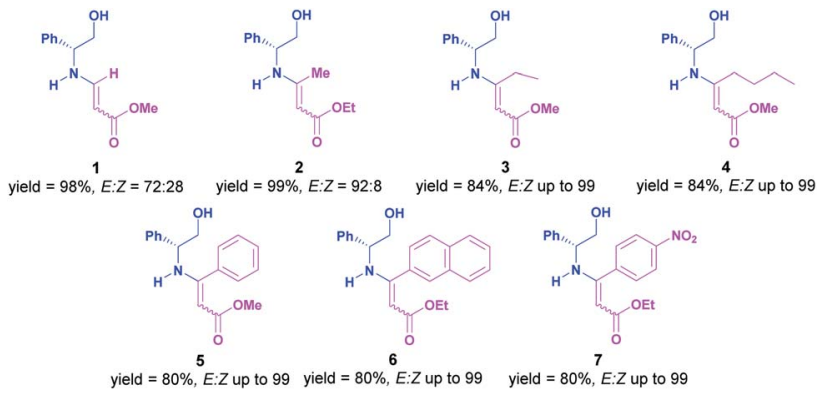

Fig. $1 \beta$-Enamino esters derived from (R)-(-)-2-phenylglycinol.

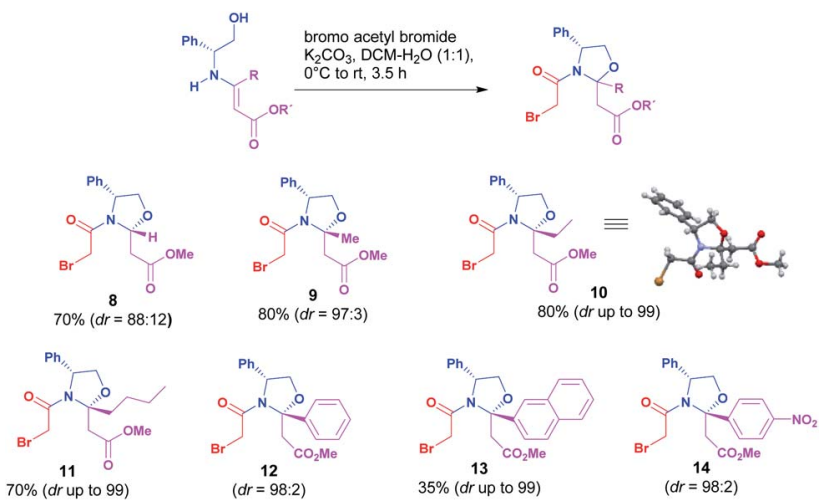

Scheme 2 Synthesis of $\mathrm{N}$-acyl oxazolidines. ${ }^{a}$ Only the major diastereoisomer is shown. ${ }^{b} \mathrm{~N}$-acyl oxazolidines 12 and 14 were probed to be unstable therefore were employed without purification for the next reaction

stereochemistry of the major diastereoisomer of each $\mathrm{N}$-acyl oxazolidine (Scheme 2).

Then, the diastereomeric mixture of $\mathbf{8}$ was selected as a model for preparing the desired zwitterionic chiral bicyclic lactam. To this end, 8 was treated with dimethyl sulfide in DCM ${ }^{11}$ delivering the corresponding sulfonium salt which was immediately subjected to the intramolecular non-classical Corey-Chaykovsky ring-closing reaction to avoid its conversion to the undesired thioether derivative.

Firstly, sulfonium salt was treated with $\mathrm{KOH}$ (2 equiv.) in a mixture of $\mathrm{CH}_{3} \mathrm{CN}: \mathrm{MeOH}(9: 1) \cdot{ }^{6}$ Unfortunately, a mixture of expected zwitterion $\mathbf{2 2}(\mathbf{a}+\mathbf{b})$ and undesired pyridine-2-one $\mathbf{2 2 c}$, was obtained. Therefore, screening experiments were performed. Gratifyingly, when the base was changed by $\mathrm{K}_{2} \mathrm{CO}_{3}$, the diastereomeric mixture of zwitterionic bicyclic lactams was exclusively obtained. ${ }^{1} \mathrm{H}$-NMR analysis of the crude reaction revealed the presence of a diastereomeric mixture of bicyclic zwitterionic intermediates in a $73: 27 d r$. Two hemiaminal protons were assigned as a doublet of doublets at $5.40 \mathrm{ppm}$ and $5.12 \mathrm{ppm}$ with coupling constants $J=9.8,5.3 \mathrm{~Hz}$ for the minor diastereoisomer and $J=11.4,4.2 \mathrm{~Hz}$ for the major diastereoisomer. The assignment was confirmed by a ${ }^{1} \mathrm{H}^{1}{ }^{1} \mathrm{H}$ COSY experiment (Table 1).

The absolute configuration at the hemiaminal position of the zwitterionic bicyclic diastereomeric mixture was
Table 1 Screening studies for nonclassical Corey-Chaykovsky ringclosing reaction $^{a}$

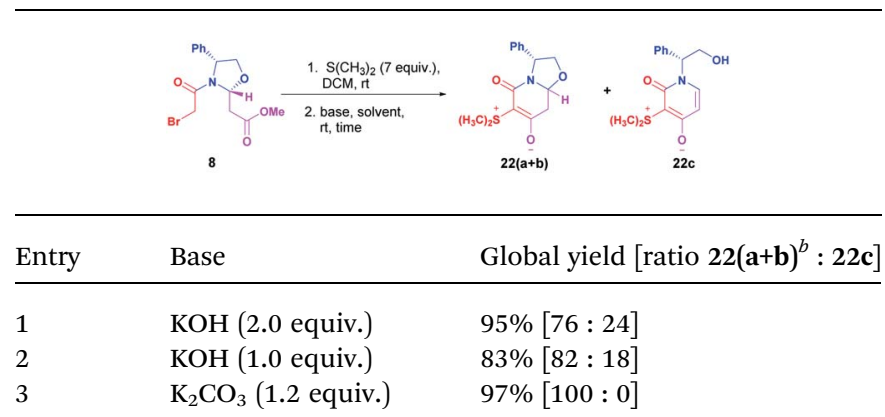

${ }^{a}$ All reactions were performed in $\mathrm{CH}_{3} \mathrm{CN}: \mathrm{MeOH}(9: 1)$, for 4 hours at room temperature. ${ }^{b} \mathbf{2 2}(\mathbf{a}+\mathbf{b})$ was obtained in a $73: 27 d r$.

determined by its X-ray diffraction analysis. A single crystal was found to include two independent molecules in the asymmetric unit of a triclinic cell (space group $P 1$ with $Z^{\prime}=2$ ), one of which presenting a disorder on the chiral center $\mathrm{C} 8 \mathrm{a}$. Combining these features results in a mixture of disatereoisomers with a ratio refined to $77.5 / 22.5 \%,{ }^{12}$ close to that determined by NMR in solution, $73 / 27 \%$. The determination of the absolute configuration in this crystal structure allowed the assignment for the major diastereoisomer 22a as $(8 \mathrm{a} R)$ and the minor diastereoisomer $\mathbf{2 2 b}$ as (8aS) (Fig. 2).

Once the optimal reaction conditions were established, the scope of our synthetic proposal was investigated. Chiral zwitterionic bicyclic intermediates containing alkyl or aryl substituents at the hemiaminal position were obtained in excellent chemical and stereochemical yields. Furthermore, the absolute configuration at the hemiaminal position was determined as $(8 \mathrm{a} R)$ for the major diastereoisomer from the X-ray diffraction analysis of zwitterions 23a and 24a (Scheme 3). ${ }^{12}$ In the case of 24a, orthorhombic single crystals feature three independent molecules in the asymmetric unit (space group $P 2_{1} 2_{1} 2_{1}, Z^{\prime}=3$ ), however, all display the same conformation and stereochemistry.

To demonstrate the utility of these new chiral zwitterionic bicyclic compounds, the diastereoselective synthesis of 4hydroxy piperidines was developed and 23a was selected as

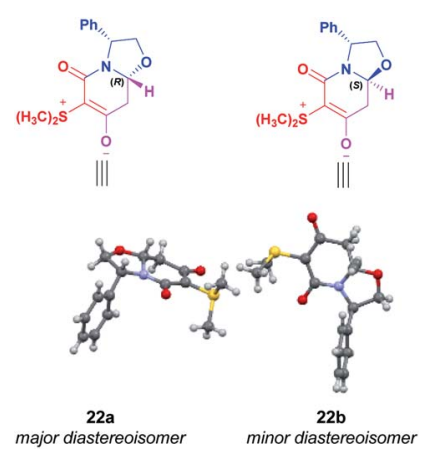

Fig. 2 X-ray structure of the diastereomeric mixture of chiral zwitterionic bicyclic intermediates $22(a+b)$. 


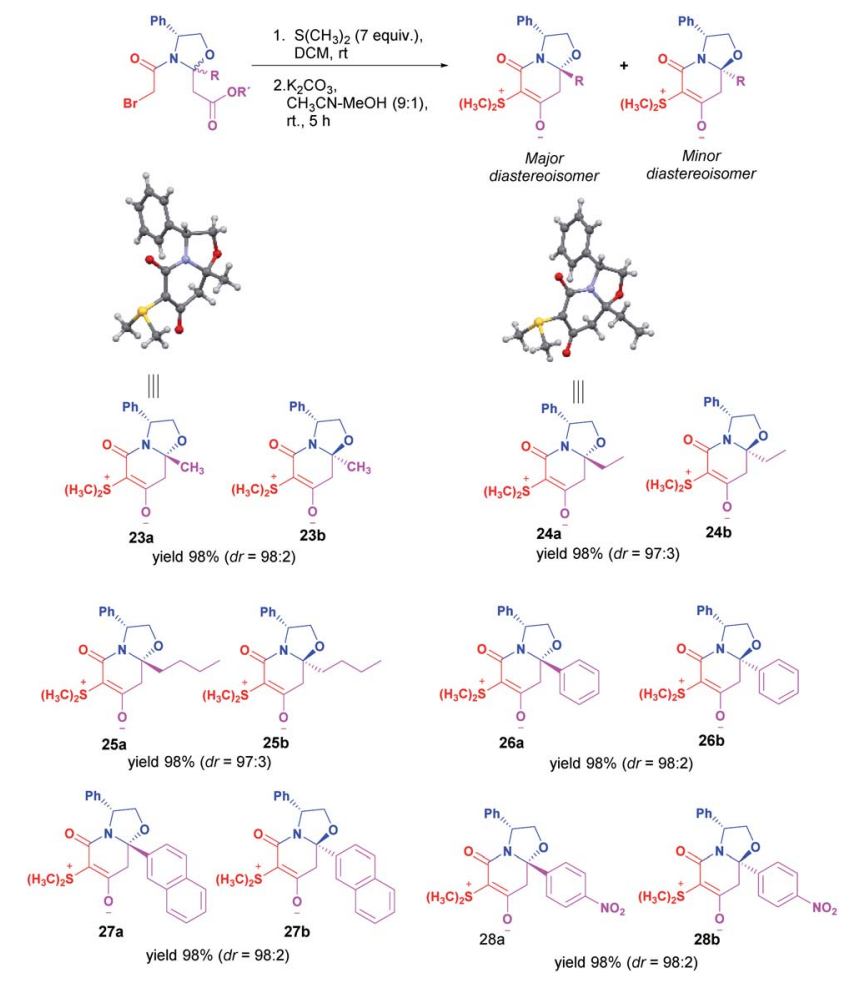

Scheme 3 Intramolecular non-classical Corey-Chaykovsky ringclosing reaction for the synthesis of chiral zwitterionic bicyclic intermediates.

a model, therefore this compound was subjected to a desulfurization process to access the corresponding oxazolo[3,2- $a]$ pyridine-5,7(6H)-dione 29. Catalytic hydrogenation was first attempted under various heterogeneous hydrogenation conditions. The use of $\mathrm{Pd}-\mathrm{C}$ or RANEY®-Ni as a catalyst in acidic or neutral conditions led the oxazolo[3,2- $a]$ pyridine-5,7(6H)-dione 29 in low chemical yield. The best result was obtained when reductive desulfurization was conducted using $\mathrm{Zn}$ (15 equiv.) in acetic acid, affording the compound 29 in $98 \%$ chemical yield
(Table 2, entry 8). In addition, compound 29 provided suitable crystals for X-ray crystallographic analysis confirming the presence of an oxazolo[3,2-a]pyridine-5,7(6H)-dione core and enabling the determination of the absolute configuration at the hemiaminal position as $(8 \mathrm{a} R),{ }^{\mathbf{1 2}}$ as in the starting material 23a.

Next, compound 29 was subjected to ketone reduction with $\mathrm{NaBH}_{4}$ (10 equiv.) in MeOH. The NMR spectrum of the crude reaction showed the presence of a diastereomeric mixture of 7hydroxy oxazolo[3,2-a]pyridin-5-one $32(\mathbf{a}+\mathbf{b})$ in an $87: 13$ diastereomeric ratio. Once the diastereomeric mixture was separated, the major diastereoisomer 32a crystallized, determining the new stereogenic center bearing the hydroxy group as $(7 R) \cdot{ }^{\mathbf{1 2}}$ The asymmetric unit of 32a contains two independent molecules (space group $P 2_{1}, Z^{\prime}=2$ ), both with the same geometry. The diastereoselectivity observed could be explained by the addition of the hydride reagent from the less hindered face, opposite to the methyl group (Scheme 4). After, reduction of the amide and hemiaminal functions of alcohol 32a was performed with $\mathrm{BH}_{3} \cdot \mathrm{DMS}$ reagent, affording the 2,4-cis-2-methyl-4-hydroxy

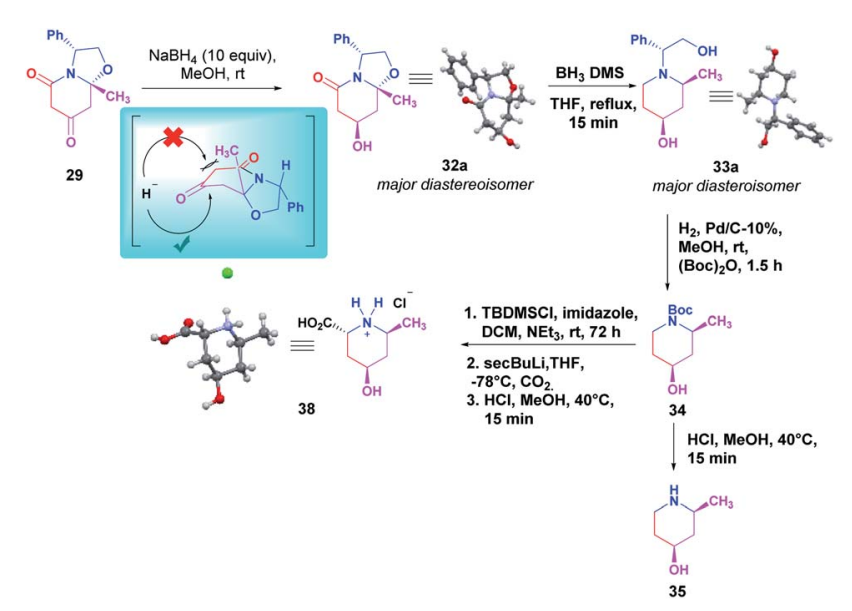

Scheme 4 Synthesis of $(2 S, 4 S)$-2-methylpiperidin-4-ol 35 and hydrochloride salt of $(2 R, 4 R, 6 S)-4$-hydroxy-6-methylpiperidine-2carboxylic acid $38 .{ }^{14}$

Table 2 Screening experiments for desulfurization of zwitterion $23 a^{a}$

\begin{tabular}{|c|c|c|c|c|c|c|}
\hline Entry & Catalyst (mol\%) & Solvent & Temp & Time & Product & Yield (\%) \\
\hline 3 & $\mathrm{Pd} / \mathrm{C}(10 \% \mathrm{~mol})$ & EtOH & Reflux & $4 \mathrm{~h}$ & $29: 30: 31$ & $38: 40: 13$ \\
\hline 4 & RANEY@-Ni & THF $: \mathrm{H}_{2} \mathrm{O}(8: 2)$ & $\mathrm{rt}$ & $8 \mathrm{~h}$ & 29 & 6 \\
\hline 5 & RANEY®-Ni & Acetone & $\mathrm{rt}$ & $14 \mathrm{~h}$ & 29 & 19 \\
\hline 6 & RANEY®-Ni & EtOH & $-10^{\circ} \mathrm{C}$ to $\mathrm{rt}$ & $8 \mathrm{~h}$ & 29 & 13 \\
\hline 7 & RANEY@-Ni & Toluene & Reflux & $8 \mathrm{~h}$ & 31 & 38 \\
\hline 8 & Zn (15 equiv.) & $\mathrm{AcOH}$ & rt & 7 days & 29 & 98 \\
\hline
\end{tabular}

${ }^{a}$ Ammonium formate was used as a hydrogen source. 
piperidine 33a as a major diastereoisomer for which the absolute configuration was unambiguously established by X-ray analysis. ${ }^{12}$ Indeed, the methyl and hydroxyl substituents are arranged cis in the piperidine ring.

Finally, complete synthesis of cis-4-hydroxy-2-methyl piperidine was accomplished by a simple debenzylation $N$-Boc protection reaction, followed by an acidic treatment to get 35 in quantitative yield. On the other hand, the synthesis of pipecolic acid derivative $\mathbf{3 8}$ was obtained through a 3-steps sequence reaction starting from intermediate 34 , which involved silyl protection of the hydroxyl function followed by a diastereoselective deprotonation-electrophilic quenching endocyclic carbonylation process to access at the pipecolic acid analog 38 . The absolute stereochemistry was assigned via the X-ray diffraction analysis of the corresponding hydrochloride salt. This compound crystallizes as a monohydrate, and the configuration $(2 R, 4 R, 6 S)^{12}$ is consistent with the refinement of a Flack parameter, $x=0.06(5)^{13}$ (Scheme 4$)$.

\section{Conclusions}

In summary, we have developed a new concise high diastereoselective strategy route to access new zwitterionic bicyclic oxazolo intermediates starting from chiral $\beta$-enaminoesters derived from $(R)-(-)$-2-phenylglycinol. In addition, the utility of these new intermediates was also demonstrated in a short diastereoselective synthesis of $(2 S, 4 S)$-2-methylpiperidin-4-ol and hydrochloride salt of $(2 R, 4 R, 6 S)$-4-hydroxy-6methylpiperidine-2-carboxylic acid. Applying this methodology to the synthesis of more complex pipecolic acids products is currently underway in our laboratory, and the results of these efforts will be reported in due course.

\section{Conflicts of interest}

There are no conflicts to declare.

\section{Acknowledgements}

We are grateful to CONACyT (Project A1-S-13280 and 268178) and VIEP-BUAP (Project 100148077-VIEP2021) for financial support. ERB thanks to CONACyT for the scholarship (658611). We thank A. L. García-Torres for HRMS spectrometry analyses.

\section{Notes and references}

1 E. Vitaku, D. T. Smith and J. T. Njardarson, J. Med. Chem., 2014, 57, 10257.

2 L. F. Roa, D. Gnecco, A. Galindo and J. L. Terán, Tetrahedron: Asymmetry, 2004, 15, 3393; L. F. Roa, D. Gnecco, A. Galindo, J. L. Terán and S. Bernès, Tetrahedron: Asymmetry, 2004, 15, 847; V. Semak, C. Escolano, C. Arróniz, J. Bosch and M. Amat, Tetrahedron: Asymmetry, 2010, 21, 2542; M. Amat, C. Escolano, N. Llor, M. Huguet, M. Pérez and J. Bosch, Tetrahedron: Asymmetry, 2003, 14, 1679; M. Amat,
M. Cantó, N. Llor and J. Bosch, Chem. Commun., 2002, 526; M. D. Groaning and A. I. Meyers, Tetrahedron, 2000, 56, 9843.

3 C. Cavé, V. Daley and J. d'Angelo, Tetrahedron: Asymmetry, 1995, 6, 79; H. M. C. Ferraz, E. O. Olveira, M. E. PayretArrua and C. A. Brandt, J. Org. Chem., 1995, 60, 7357; C. J. Valduga, H. S. Braibante and E. F. J. Braibante, J. Heterocycl. Chem., 1998, 35, 189; A. Amougay, O. Letsh and J. P. Pete, Tetrahedron, 1996, 52, 2405; C. Cimarelli and G. Palmieri, J. Org. Chem., 1996, 61, 5557; S. Ali and A. T. Khan, Tetrahedron Lett., 2013, 54, 436.

4 P. W. Hickmott and G. Sheppard, J. Chem. Soc. C, 1971, 1358; P. W. Hickmott and G. Sheppard, J. Chem. Soc. C, 1971, 2112; C. Agami, L. Dechoux and S. Hebbe, Tetrahedron Lett., 2003, 44, 5311; C. Agami, L. Dechoux, C. Ménard and S. Hebbe, J. Org. Chem., 2002, 67, 7573; C. Agami, L. Dechoux, S. Hebbe and C. Ménard, Tetrahedron, 2004, 60, 5433.

5 R. López-González, A. Zarate, D. M. Aparicio, A. Mendoza, D. Gnecco, J. R. Juárez, N. Romero-Ceronio, L. Orea and J. L. Terán, Tetrahedron Lett., 2016, 57, 1683.

6 R. López-González, D. Gnecco, J. R. Juárez, V. GómezCalvario, S. Bernès, D. M. Aparicio and J. L. Terán, Tetrahedron Lett., 2020, 61, 151697.

7 D. F. Martin, G. A. Janusonis and B. B. Martín, J. Am. Chem. Soc., 1961, 83, 73; B. Rechsteiner, F. Teixier-Boullet and J. Hamelin, Tetrahedron Lett., 1993, 34, 5071; A. Arcadi, G. Bianchi, S. Di Giuseppe and F. Marinelli, Green Chem., 2003, 5, 64; A. R. Khosropour, M. Khodaei and M. Kookhazadeh, Tetrahedron Lett., 2004, 45, 1725; Z. H. Zhang, L. Yin and Y. M. Want, Adv. Synth. Catal., 2006, 348, 184; R. Dalpozzo, A. Deino, M. Nardi, B. Russo and A. Procopio, Synthesis, 2006, 1127; J. Lin and L. F. Shang, Monatsh. Chem., 2007, 138, 77; F. Epofano, S. Genovese and M. Curini, Tetrahedron Lett., 2007, 48, 2717.

8 B. J. Turunen and G. I. Georg, J. Am. Chem. Soc., 2006, 128, 8702; N. Panda and R. Mothkuri, J. Org. Chem., 2012, 77, 9407; H. Pilotzi, D. Gnecco, M. L. Orea, J. R. Juárez, D. M. Aparicio and J. L. Terán, Heterocycles, 2018, 96, 895.

9 D. M. Aparicio, D. Gnecco, J. R. Juárez, M. L. Orea, N. Waksman, R. Salazar, M. Flores-Alamo and J. L. Terán, Tetrahedron, 2012, 68, 10252.

10 CCDC: 2125364 (10), contain the ESI crystallographic data for this paper. $\dagger$

11 D. M. Aparicio, J. L. Terán, D. Gnecco, A. Galindo, J. R. Juárez, M. L. Orea and A. Mendoza, Tetrahedron: Asymmetry, 2009, 20, 2764.

12 CCDC: 2125365 (22(a+b)), 2125366 (23a), 2125367 (24a), 2125368 (29), 2125369 (32a), 2125370 (33a), 2125371 (38) contain the $\mathrm{ESI}^{\dagger}$

13 H. D. Flack and G. Bernardinelli, Acta Crystallogr. Sect. A, 1999, 55, 908.

14 In this article, Authors reported the corresponding pipecolic acid enantiomer: L. S. Fowler, L. H. Thomas, D. Ellis and A. Sutherland, Chem. Commun., 2011, 47, 6569. 